\title{
La formación del docente en relación a la diversidad y educación inclusiva
}

\section{Teacher training in relation to diversity and inclusive education}

1 Mirella Margarita Gaibor Sangache $\quad$ (iD) https://orcid.org/0000-0002-3232-980X

Universidad Técnica de Ambato, Facultad de Ciencias Humanas y de la Educación,

Carrera de Psicopedagogía Tungurahua

mgaibor2007@uta.edu.ec

2 Diana Alexandra Machay Negrete (iD) https://orcid.org/0000-0001-7300-4940

Universidad Técnica de Ambato, Facultad de Ciencias Humanas y de la Educación,

Carrera de Psicopedagogía Tungurahua

dmachay5639@uta.edu.ec

3 María Belén Morales Jaramillo (iD) https://orcid.org/0000-0003-4534-3480

Universidad Técnica de Ambato, Facultad de Ciencias Humanas y de la Educación,

Tungurahua,

mb.morales@uta.edu.ec

4 Jorge Rodrigo Andrade Albán (iD) https://orcid.org/0000-0002-7389-6624

Universidad Técnica de Ambato, Facultad de Ciencias Humanas y de la Educación,

Tungurahua

jr.andrade@uta.edu.ec

Artículo de Investigación Científica y Tecnológica

Enviado: 24/12/2021

Revisado: 29/12/2021

Aceptado: $12 / 01 / 2022$

Publicado:08/03/2023

DOI: https://doi.org/10.33262/concienciadigital.v6i1.4.2009

Cítese:

Ciencia

Digital

(1) (2)

(4.0
Gaibor Sangache, M. M., Machay Negrete, D. A., Morales Jaramillo, M. B., \& Andrade Albán, J. R. (2023). La formación del docente en relación a la diversidad y educación inclusiva.

ConcienciaDigital,

6(1.4),

474-488.

\section{https://doi.org/10.33262/concienciadigital.v6i1.4.2009}

\footnotetext{
CONCIENCIA DIGITAL, es una Revista Multidisciplinar, Trimestral, que se publicará en soporte electrónico tiene como misión contribuir a la formación de profesionales competentes con visión humanística y crítica que sean capaces de exponer sus resultados investigativos y científicos en la misma medida que se promueva mediante su intervención cambios positivos en la sociedad. https://concienciadigital.org

La revista es editada por la Editorial Ciencia Digital (Editorial de prestigio registrada en la Cámara Ecuatoriana de Libro con No de Afiliación 663) www.celibro.org.ec

Esta revista está protegida bajo una licencia Creative Commons Attribution Non Commercial No Derivatives

4.0 International. Copia de la licencia: http://creativecommons.org/licenses/by-nc-nd/4.0/
} 
Palabras

claves:

inclusión

educativa, diversidad, políticas, formación docente.

Keywords: educational inclusion, diversity, policies, teacher
Resumen

Introducción. Este artículo tiene como finalidad comprender el desafío de la educación actual centrada hacia la concepción de la diversidad e inclusión conforme a los ejes de justicia e inclusión establecida por la sociedad. Un sistema educativo el cual promueva la atención sin discriminación y priorizando el respeto a sus derechos. Tomando como punto de partida al enfoque de la formación docente, como instauradores principales de inclusión dentro del aula y el centro formativo, aplicando un trabajo dirigido a la atención de las diversidades. Objetivo. Con el fin de conocer diferentes perspectivas de los docentes hacia la diversidad y educación inclusiva, además, identificar el nivel de formación y conocimientos que adquieren en el transcurso profesional. Metodología. La obtención de datos se realizó a través del cuestionario formación docente en inclusión de profesionales de la educación -CEFI-R. Se tomó una muestra de 65 docentes de la Universidad Técnica de Ambato. Resultados. Los resultados en las 4 dimensiones planteadas, dimensión de concepción de diversidad el $69 \%$ muestran actitudes positivas hacia la diversidad, dimensión metodológica determina que el $72 \%$ maneja una metodología adecuada, dimensión de apoyo evidencian que el $69 \%$ de los docentes proporcionan apoyos a sus alumnos y dimensión de participación de la comunidad el $86 \%$ establece el trabajo colaborativo entre el centro escolar, sus agentes, familia y la sociedad. Conclusión. Se ha concluido que la formación docente dentro de la Universidad Técnica de Ambato está encaminada al desarrollo de una educación que valore, respete las diferencias y enriquezca el proceso de enseñanza-aprendizaje. Sin embargo, promover el ejercicio de la educación inclusiva a nivel general es complejo puesto que el cantón Ambato se encuentra en proceso. Pocas han sido las instituciones interesadas en establecer escuelas inclusivas.

\section{Abstract}

Introduction. This article aims to understand the challenge of current education focused on the conception of diversity and inclusion according to the axes of justice and inclusion established by society. An educational system which promotes care without discrimination and prioritizing respect for their rights. Taking as a starting point the approach to teacher training, as main instigators 
training. of inclusion within the classroom and the training center, applying a work aimed at addressing diversity. Target. To know different perspectives of teachers towards diversity and inclusive education, in addition, identify the level of training and knowledge they acquire in the professional course. Methodology. Data was obtained through the teacher training questionnaire on the inclusion of education professionals -CEFI-R. A sample of 65 teachers from the Technical University of Ambato was taken. Results. The results in the 4 proposed dimensions, dimension of conception of diversity $69 \%$ show positive attitudes towards diversity, methodological dimension determines that $72 \%$ use an adequate methodology, support dimension shows that $69 \%$ of teachers provide support to their students and community participation dimension $86 \%$ establish collaborative work between the school, its agents, family, and society. Conclusion. It has been concluded that teacher training within the Technical University of Ambato is aimed at developing an education that values, respects differences, and enriches the teaching-learning process. However, promoting the exercise of inclusive education at a general level is complex since the canton of Ambato is in the process. Few have been the institutions willing to establish inclusive schools.

\section{Introducción}

En los últimos años los termino de "diversidad" e "inclusión" han ido tomando relevancia en el ámbito educativo a nivel mundial y nacional, además se encuentra inmerso en diferentes ámbitos como las políticas públicas y las distintas acciones gubernamentales.

La diversidad alude al término "propio" es decir, es la identificación del ser humano como es y no como la sociedad espera que sea (Rubiales, 2010). Comprender la perspectiva de lo diverso hace único a cada individuo. De este modo asegurarse que a una institución educativa ingresan alumnos con las mismas características es algo complejo de afirmar ya que cada individuo posee sus cualidades culturales, sociales y económicas propias, señalando la diversidad como tal, desde esta perspectiva se debe considerar un reto dentro del sistema educativo, dando prioridad al trabajo de la inclusión.

Políticas gubernamentales como la (Organización de las Naciones Unidas para la Cultura, las Ciencias y la Educación [UNESCO], 2009) contempla que la educación inclusiva es considerada una estrategia importante para lograr la Educación de calidad para todos, dicha declaración se adquirió desde 1990, basada en el hecho de que la educación es un 
derecho. Es decir, son derechos humanos fundamentales y básicos para una sociedad más justa e igualitaria.

A través de la UNESCO, y la Declaración sobre necesidades educativas especiales: Acceso y calidad, representa un importante compromiso suscrito por varios líderes de diferentes países con la inquebrantable intención de brindar educación a todos los estudiantes que anteriormente se encontraban sujetos a circunstancias de exclusión del sistema educativo. Dicha declaración fundamentada principalmente en atender las necesidades educativas especiales (NEE), es decir que sus ventajas más importantes radican en su éxito en la implantación de la educación universal a nivel internacional y en la promoción del desarrollo de cambios en los sistemas y políticas educativas, y en el mantenimiento de la viabilidad de estos métodos en todos estos años. Una educación para todos especialmente para aquellos grupos más vulnerables a la exclusión, la marginación y la discriminación.

Para ello, el Ecuador también ha implementado acciones, a través del Plan Nacional del Buen Vivir, en conformidad con el objetivo 2, busca "Auspiciar la igualdad, la cohesión, la inclusión y la equidad social y territorial, en la diversidad", dentro de este marco la política. Fomentar la inclusión y cohesión social, la convivencia pacífica y la cultura de paz, erradicando toda forma de discriminación y violencia.

Por lo dicho, para dar prioridad a la diversidad se establece en su artículo 341 de la Constitución de la República del Ecuador (2008):

Asegurar el cumplimiento de los derechos y principios de igualdad en la diversidad y la no discriminación, a aquellos grupos en el que muestran desigualdades, exclusión, discriminación o violencia, o en virtud de su condición etaria, de salud o de discapacidad.

En consideración a lo mencionado el estado ecuatoriano es veedor de la protección de derechos en cumplimiento de la atención a las necesidades educativas, quien promulga en las instituciones educativas el cumplimiento de las políticas regidas para su desarrollo y garantizan calidad educativa.

La inclusión un proceso dinámico y complejo a través del cual puede integrar a todos los estudiantes con o sin necesidades educativas especiales al contexto educativo. La integración se ha asociado con las personas con discapacidades o las necesidades educativas especial, lo cual existen exclusión por parte de los compañeros del aula de clases al considerar que no son iguales a ellos, con las mismas capacidades, es decir existe una carga ideológica que delimita el acceso de inferioridad por consiguiente la exclusión. Las responsabilidades de las instituciones educativas y en especial los docentes 
actualmente están implementando reformas, adaptación educativa y programas específicos para de este modo tratar de contrarrestar ciertas diferencias (Duk et al., 2019).

La formación docente es fundamental para un cambio en la sociedad, que valore el desarrollo y los proyectos de vida humana, por lo que diversos procesos pedagógicos se conviertan en una meta constante y deseada, requeridos por la cultura del desarrollo como sujetos. Como señala Muntane et al. (2016) "aprender a trabajar en los centros educativos con la diversidad del alumnado, manteniendo la igualdad de oportunidades y las altas expectativas de éxito para todos los estudiantes, representa los dos grandes desafíos del modelo educativo actual" (p. 46). Por tanto, en el marco de inclusión en el campo de la educación, además de las competencias básicas integrales, trasversales instrumentales, interpersonales, la comunicación entre personas y sistemas necesarios para cualquier profesional de calidad y competitivo, un conjunto de competencias que se requieren, de una manera u otra.

La necesidad de nuevos enfoques de la formación docente que priorice, su visión como objetos de aprendizaje activo, su crecimiento personal y de largo plazo, así como agentes de educación y orientación pedagógica para sus alumnos, que se puede asumir a partir de la perspectiva de Vygotsky asociados en conocer las potencialidades de desarrollo del docente siendo esta la zona de desarrollo próximo según su autor. Esto significa abordar un nuevo concepto de formación docente y algunos de sus componentes (Nieva \& Martínez, 2016).

La aspiración del sistema educativo a nivel nacional e internacional es capacitar a los docentes para que trabajen de manera adecuada con una diversidad de alumnos en un contexto de aulas inclusivas, en la cual promueva la equidad y la justicia social. En este sentido, la Agenda 2030 con el fin de avanzar hacia sociedades con un gran crecimiento económico, desarrollo social, justicia, siendo más incluyentes, en paz y con un horizonte ambiental sostenible, promoviendo de este modo el bienestar colectivo. Dicha agenda destaca la necesidad de: garantizar una educación de calidad con base inclusiva, equitativa y promover oportunidades de aprendizaje permanente para todos sin ningún tipo de exclusión (Naciones Unidas, 2018).

Asimismo, lograr un sistema educativo centrado en la diversidad depende no únicamente del trabajo del sistema educativo sino de la labor docente. En ese sentido la formación que este adquiere en la educación superior debe enfocarse en criterio de formación de atención hacia la diversidad propiciando la inclusión, por lo tanto, las instituciones universitarias son entornos formativos donde se debe propiciar iniciativas en la educación en la diversidad, interculturalidad e inclusión (Zapata, 2016).

Marchesi et al. (2009) refiere que la buena práctica integral debe entenderse como un acto de 'determinar', que es lógico y posible a partir de una realidad dada, a partir de algún 
factor estructural adaptativo que la haga única e insustituible. No existen las mejores prácticas ideales porque depende del contexto en el que se desarrolle.

Basándose en un enfoque inclusivo con atención hacia la diversidad ha permitido reconocer las problemáticas dentro del sistema, para ejemplificar, los docentes no se están totalmente capacitados para atender este tipo de necesidades de los alumnos, y la organización de dichos proceso de inclusión tanto a nivel institucional y áulico no es acorde, al no disponer de los recursos necesarios para implementar adaptaciones o soporte de ayuda para padres y en otros casos es porque el número de actividades que está obligados a cumplir el docente dentro de su labor el tiempo no le es suficiente para cumplir con estos requerimientos.

No obstante, otros estudios declaran que enseñar a diversidades puede llegar a ser complejo y son percibidos como un problema (Ruiz, 2019). Sin embargo, viéndolo desde un punto de vista positivo, este contexto permite además de crear nuevas interacciones, crear en el alumno el sentido de diversidad como una forma de aprendizaje y autoconciencia, en el que aporten nuevas maneras de concebir el aprendizaje en diversidades.

Ofrecer a la sociedad una educación de calidad exige como objetivo del docente, proveer a sus educandos las competencias necesarias para desempeñarse autónomamente dentro de la sociedad (Herreros, 2016). De acuerdo ello según Rivadeneira Ferrín (2012) "el perfil debe orientarse a la formación de profesionales conocedores de su identidad pluri étnica cultural, capaz de fomentar competencias que valoren, respeten y acepten la diversidad en las actividades educativas y socioculturales" (pág. 50).

Watkins (2012), determina cuatro valores en la enseñanza y el aprendizaje que sustenta el trabajo de todos los docentes en la educación inclusiva. Se detalla a continuación además las distintas áreas de competencia que influyen en el docente:

1. Valorar positivamente la diversidad de los alumnos. - Las distintas concepciones de la educación inclusiva y opiniones que proporcionan los docentes sobre las distintas diferencias.

2. Apoyar a todos los alumnos. - Con base a las diferentes áreas de competencias, promoviendo de este modo el aprendizaje tanto practicó, emocional y social de todos los alumnos además de los enfoques metodológicos aplicados dentro del aula de clases.

3. Trabajo en equipo. - se refiere que al trabajo aplicado con un amplio número de profesionales de la educación y con las familias.

4. Desarrollo profesional y personal. - Los docentes siendo profesionales con capacidad de reflexión y la formación de los docentes con base en el aprendizaje y desarrollo profesional continuo. 
Los factores mencionados, siempre van a ir acompañados de las áreas de desarrollo, donde Watkins cita que estas áreas se componen de tres elementos: actitudes, conocimientos y habilidades. Debido a que se requiere un nivel de conocimiento y comprensión, por lo tanto, se requieren habilidades para aplicar ese conocimiento en situaciones de la vida real.

Por tanto, todos los factores y áreas deben ser considerados como base para desarrollar itinerarios formativos y como punto de partida para la realizando de debates de diferentes niveles sobre el contexto específico de las diferentes áreas que se desenvuelve el docente (Watkins, 2012).

Por otra parte, Colina (2017) menciona algunas de las competencias que el docente debe adquirir: competencia interpersonal; competencia metodológica; estrategias metodológicas; habilidad comunicativa; competencia de planificación y gestión de la docencia; trabajo en equipo: competencia de innovación (pág. 39).

La flexibilidad es además una característica del docente ya que además de adquirir dichas competencias, este debe dar la posibilidad de innovar sus metodologías de enseñanzaaprendizaje, dando prioridad a las necesidades suscitadas dentro del aula.

De acuerdo Duk et al., (2019), los desafíos para iniciar una formación docente en las cuales infieren esta política son:

- Entender el fenómeno de la discapacidad desde otro enfoque multidimensional como psicosocial y desarrollar habilidades para identificar y evaluar de manera oportuna las NEE, los planes de estudio y los enfoques pedagógicos.

- Desarrollar la capacidad de pensar críticamente sobre los dilemas de inclusión y las tensiones que surgen de las inconsistencias en los enfoques, políticas y prácticas.

- Promover el desarrollo de las habilidades comunicativas, sociales, cognitivas y emocionales necesarias para trabajar en entornos colaborativos, para promover el aprendizaje y la participación de todos los estudiantes.

- Fomentar el uso de una variedad de métodos de enseñanza, estrategias de aprendizaje y apoyo que permitan a todos tomar decisiones que mejoren el aprendizaje, teniendo en cuenta sus diferencias.

Cabe recalcar, que dentro de la sociedad cada vez hallan mayores exigencias por lo que las competencias docentes deben de ser reforzadas constantemente, lo cual permite actuar en consecuencia de la situación es decir induciendo a nuevas prácticas metodológicas acorde a la dificultad.

Por lo mismo, La formación del docente debe ser regular y continua, para convertirse en un verdadero agente de cambio social. Se necesita de nuevos conocimientos sobre sus 
aspectos conceptuales más generales en el contexto actual, donde el conocimiento pragmático, instrumentalista y tecnológico sea amplio para el desarrollo humano del docente en un proceso de significación personal y social (Nieva \& Martínez, 2016).

Otras investigaciones validan la importancia de las actitudes docentes frente a las diversidades ya que como menciona Alemany \& Villuendas (2004) "mantener una actitud abierta hacia la diversidad es una de las claves para el nuevo campo educativo y su trasformación", una adecuada formación y ejecución de habilidades y respuestas permite mantener seguridad al momento de afrontar retos educativos y por consiguiente demostrar actitudes favorables y fundamentales en el trabajo con necesidades educativas recalcando su labor hacia el trabajo diversificado.

Según Sarrionandía \& Ainscow (2011) manifiestan cuatro elementos importantes de la inclusión:

- La inclusión un proceso. En otras palabras, la inclusión debe ser vista como una búsqueda constante de mejora en cuanto a la diversidad de todos los estudiantes. Bajo esta presuposición "Aprende a vivir con las todas sus diferencias y aprende al mismo tiempo bajo cualquier condición".

- La inclusión busca participación, presencia y éxito para todos los alumnos, incluir la búsqueda proceso de enseñanza-aprendizaje haciéndole participe en todo momento al alumno.

- La inclusión la identificación precisa y la eliminación de todas las barreras que pudieran existir, se considera barreras al interactuar con las demás personas con diferentes condiciones tanto en el ámbito social, cultura y personal.

- La inclusión se enfoca específicamente en grupos de estudiantes que pueden estar en riesgo de marginación, exclusión o fracaso académico.

Dentro de este marco se debe involucrar a todas las partes interesadas considerando la comunidad local, incluyendo a las familias, religiosos, líderes políticos y los medios de comunicación. Lógicamente, se debe involucrar a los responsables de la gestión del sistema educativo en el ámbito tanto en el contexto local o regional.

En definitiva, conocer el grado de formación de los docentes en cuanto la diversidad e inclusión y además de algunas de los desafíos que enfrentan los docentes en las aulas de clases. La formación de docentes en inclusión educativa requiere el encuentro de amplios contextos sociales para diseñar programas que aborden múltiples posibilidades con el fin de desarrollar todas las competencias educativas necesarias para el desarrollo humano. En este sentido, el docente necesita tener un conocimiento amplio del contexto educativo, además de poseer habilidades que le permitan al estudiante desarrollar plenamente sus capacidades y potencialidades. 


\section{Metodología}

El estudio de la formación del docente en relación con la diversidad y educación inclusiva se orienta hacia un grupo de docentes que imparten su enseñanza en la Universidad Técnica de Ambato, de la facultad de ciencias humanas y de la educación, ubicada en la Provincia de Tungurahua.

Para la recopilación de los datos se tomó como referencia el cuestionario desarrollado y validado para evaluar la formación docente en inclusión de profesionales de la educación -CEFI-R- con un contenido de dieciséis preguntas, las cuales se encuentran divididas en cuatro dimensiones (Concepción de la diversidad; metodología; apoyos y participación de la comunidad). Dicho cuestionario está conformado por preguntas basadas en una escala de Likert, en donde cada una de las preguntas poseen cinco valores que van de un rango 0 al 4. Con la información obtenida se contará con datos numéricos y exactos en cuanto al nivel de preparación y formación docente con respecto a la enseñanza inclusiva de una manera deductiva (Hernández et al., 2004).

\section{Resultados}

Se seleccionaron docentes de los diferentes niveles de formación. De este modo, la muestra estuvo formada por un total de 65 docentes, con un rango de edad aproximadamente entre los 25 años a los 46 años, dividida de la siguiente manera; 43 mujeres y 22 hombres. Al realizar un análisis combinado entre el enfoque cuantitativo y cualitativo obteniendo como resultado un enfoque mixto. De acuerdo con Sampieri et al. (2016) manifiesta: "Los métodos de investigación mixta es aquella integración sistemática tanto del método cuantitativo y cualitativo en un solo estudio con el fin de obtener una perspectiva más completa del fenómeno" (p.156).

\section{Figura 1}

\section{Población}

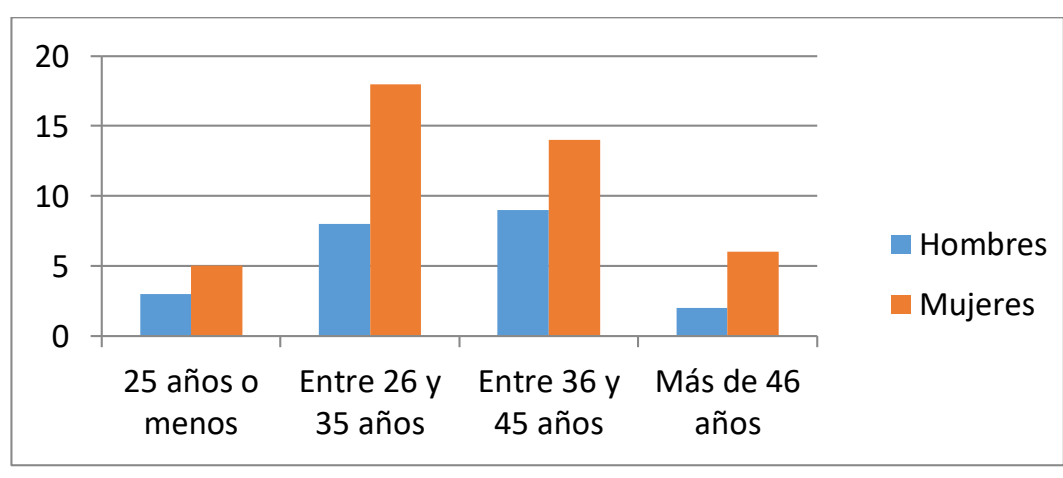

Nota: Número de docentes encuestados divididos, por rango de edad y géneros con la numeración respectiva de los encuestados. 


\section{Figura 2}

Años de Experiencia Docente

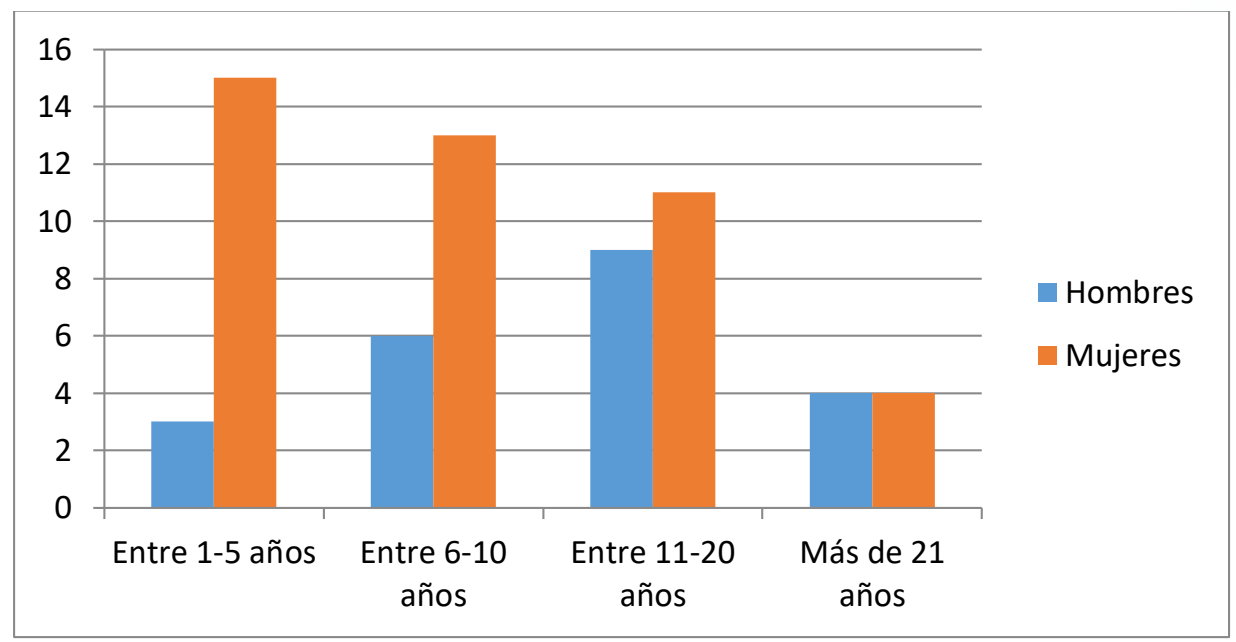

Nota: Evidencia el número de encuestados divididos en hombres y mujeres, de acuerdo con los de experiencia docente.

Como podemos evidenciar en la figura 3 , tomando en consideración que 0 mantiene una interpretación positiva y el 4 negativa se ha determinado que el $33 \%$ y $36 \%$ con determinante positiva, aseguran que los profesores integran, atienden y toman en consideración al trabajo con niños con Necesidades Educativas Especiales.

\section{Figura 3}

\section{Concepción de Diversidad}

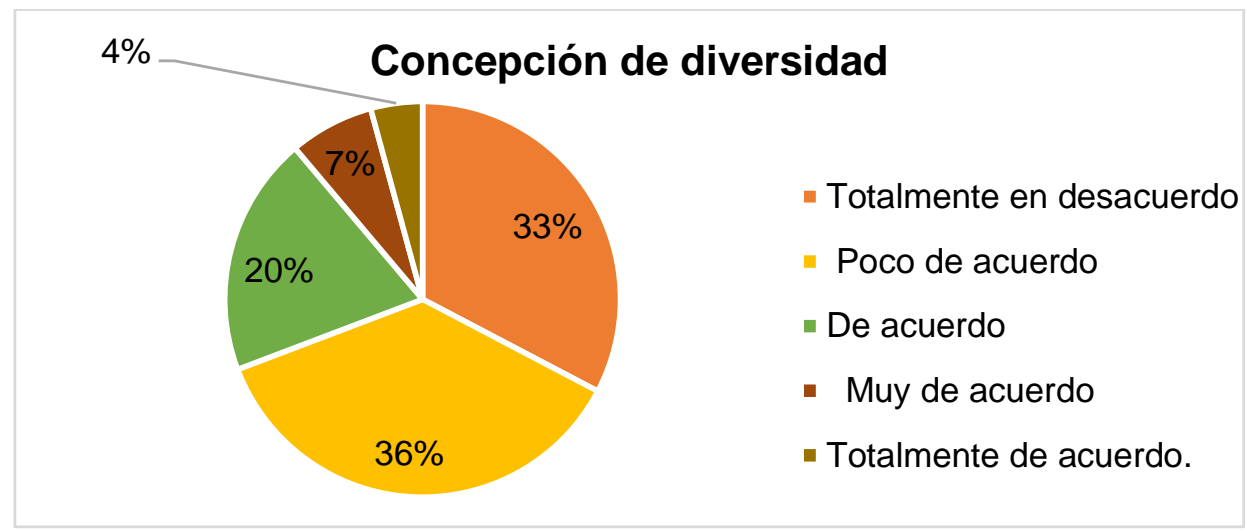

Nota: Valoración de las perspectivas ideológicas en cuanto la diversidad.

Como podemos ver en la figura 4 , señalando que el 4 es el puntaje positivo y 0 es un puntaje negativo, se determina que el $19 \%$ y el $53 \%$ de la población conoce, desarrolla, adapta metodologías pertinentes acorde a la necesidad del alumno. 


\section{Figura 4}

Dimensión Metodológica

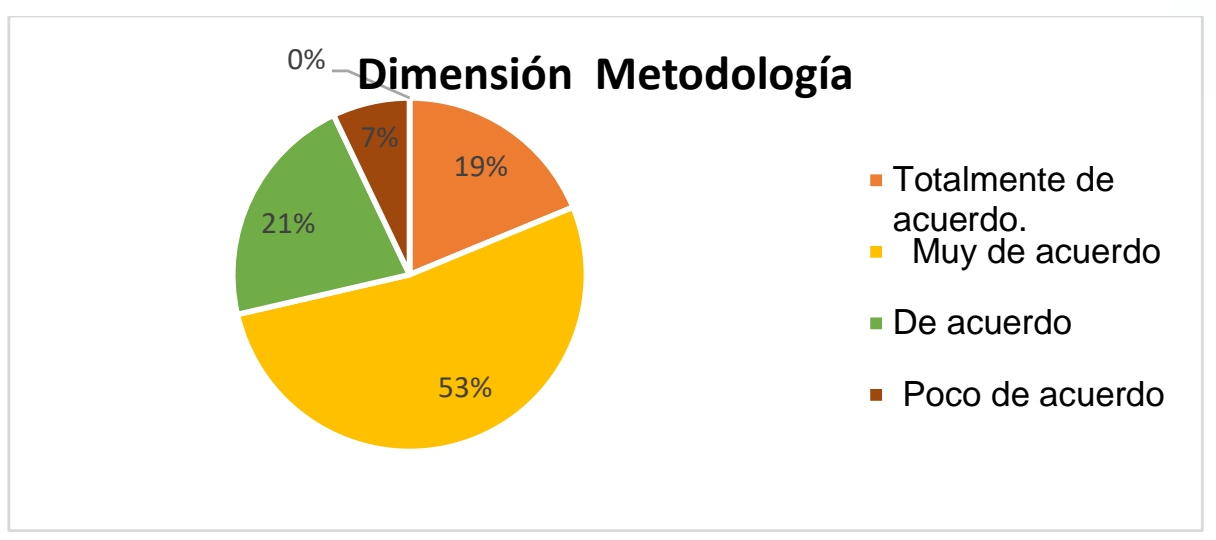

Nota: Evaluación de la aplicación de metodologías inclusivas.

Con respecto a la figura 5, los resultados del cuestionario aplicado a 65 docentes evidencian que el $44 \%$ y $25 \%$ de los docentes creen que la mejor manera de proporcionar apoyos a los alumnos es que el profesor de apoyo se incorpore al aula, respalde en la planificación, trabajo. Determinando así, que el trabajo en conjunto con ambos actores favorece la ejecución y desarrollo hacia el grupo y el alumno con necesidades.

\section{Figura 5}

\section{Dimensión Apoyos}

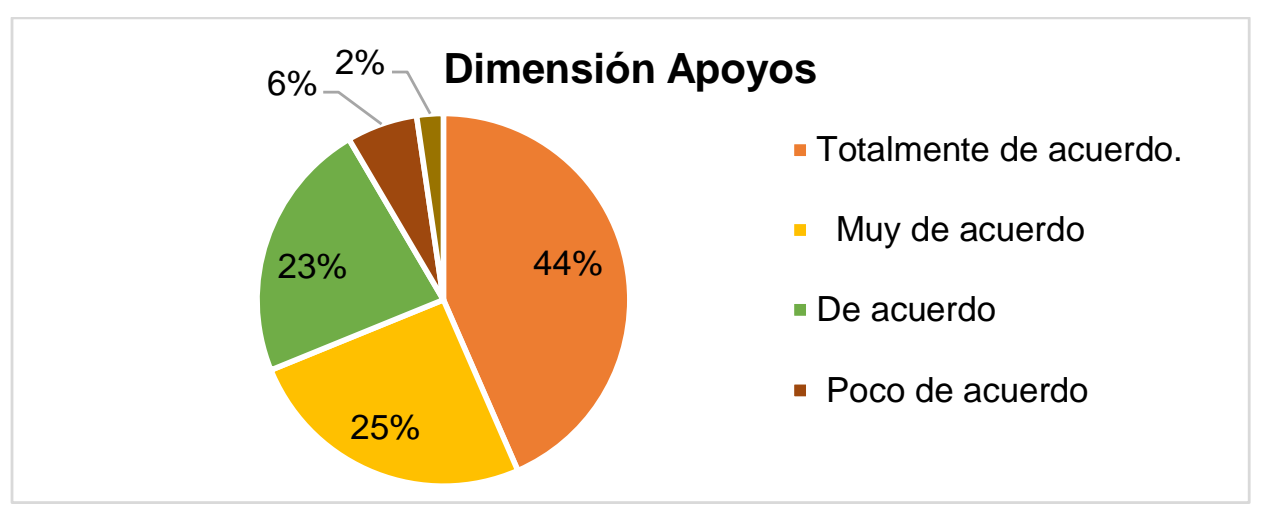

Nota: Valoración del apoyo personal ejercido hacia el docente y estudiantes.

Tomando en consideración a la figura 6, el 67\% y 19\% indican que la población estudiada reconoce la importancia de la participación del centro escolar, sus agentes, familia, y la sociedad, para propiciar el desenvolvimiento del estudiante dentro y fuera de ella. 


\section{Figura 6}

Dimensión Participación de Comunidad

\section{Dimensión Participación de la Comunidad}

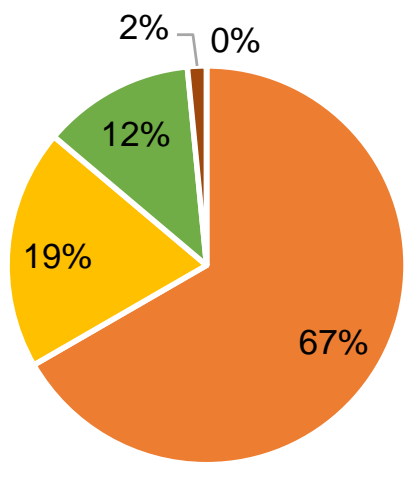

- Totalmente de acuerdo.

- Muy de acuerdo

- De acuerdo

- Poco de acuerdo

- Totalmente en desacuerdo

Nota: Estimación de niveles de actuación de los distintos contextos con aporte al desarrollo del estudiante.

\section{Conclusiones}

- Tras el análisis, varios han sido los aspectos en los cuales debe enfocase el proceso de formación docente como la inclusión, en cual muestra actitudes que apoyan la adquisición los métodos de enseñanza-aprendizaje, profesionales capaces de diseñar programas que aborden múltiples posibilidades con el fin de desarrollar todas las competencias educativas necesarias para el crecimiento del alumno. Considerando a las experiencias vividas como traductor de recomendaciones para la formación docente inclusiva.

- De esta forma, las dimensiones evaluadas han sido un camino esencial para identificar las perspectivas hacia la diversidad e inclusión, el mismo que permitió establecer que en su mayoría los docentes están predispuesto a trabajar con estudiantes con o sin necesidad educativa especiales, puesto que no impiden el aprendizaje de sus compañeros y así favorecen el desarrollo de aulas inclusivas, razón por la cual reconocer que cada individuo posee sus propias capacidades y potencialidades abre paso a la interacción y sobre todo la inclusión. Actualmente los docentes conocen más acerca de las metodologías de enseñanza. En este sentido, la capacidad de elaborar y planificar unidades didácticas e incluso evaluar tomando en consideración la diversidad que existe en su aula de clase y principalmente priorizando sus necesidades.

- Finalmente, la implementación de la inclusión no es únicamente el papel u obligación del docente o del director, sino que este abarca un campo amplio en el 
que cada uno de los actores educativos tienen un papel fundamental en el desarrollo del estudiante, de esta manera como se ha venido evidenciando en la bibliografía es fundamental la relación que debe existir entre los profesores, padres de familia y la sociedad en sí, ya que hacer la praxis de la inclusión es tarea del sistema educativo y de la sociedad.

\section{Referencias bibliográficas}

Alemany, I., \& Villuendas, M. (2004). Las actitudes del profesorado hacia el Alumno con Necesidades Educativas Especiales. Convergencia. Revista de Ciencias Sociales, 183-215.

Colina, A. (2017). Competencias docentes en la enseñanza de la educación superior Ecuador. Samborondón: II Congreso Científico Internacional.

Constitución de la Republica del Ecuador. (2008, 20 de octubre). Articulo 341 [Titulo VII]. Diario oficial de la Federación. Obtenido de https://www.oas.org/juridico/pdfs/mesicic4_ecu_const.pdf

Duk, C., Cisternas, T., \& Ramos, L. (2019). Formación docente desde un enfoque inclusivo. A 25 años de la Declaración de Salamanca, nuevos y viejos desafíos. Revista latinoamericana de educación inclusiva, 13(2), 91-109.

Hernández, S., Fernández-Collado, R., \& Baptista, C. (2004). Metodología de la Investigación. México: McGraw-Hill Interamericana.

Herreros, L. (2016). La formación del docente ante la diversidad en el aula [Tesis de Maestría, Universidad Rey Juan Carlos]. Obtenido de http://riberdis.cedd.net/bitstream/handle/11181/5675/La_formaci\%F3n_del_doc ente_ante_la_diversidad_en_su_aula.pdf;jsessionid=FC26E924386E715CB8233 D4E208EEF28? sequence $=1$

Marchesi, Durán, Climent, \& Hernández. (2009). Guía para la reflexión y valoración de prácticas inclusivas. Madrid.

Muntaner, J., Rosselló, M., \& De la Iglesia, B. (2016). Buenas prácticas en educación inclusiva. Educación siglo XXI, 34(1), 31-50.

Naciones Unidas. (2018). La Agenda 2030 y los Objetivos de Desarrollo Sostenible: una oportunidad para América Latina y el Caribe. Santiago.

Nieva, J., \& Martínez, O. (2016). Una nueva mirada sobre la formación docente. Revista Universidad y Sociedad, 8(4), 14-21. 
Organización de las Naciones Unidas para la Cultura, las Ciencias y la Educación [UNESCO]. (2009). Organización de las Naciones Unidas para la Educación, la

Rivadeneira Ferrín, E. (2012). Perfil de competencias de la formación docente en los Institutos Superiores Pedagógicos del País [Tesis de Maestría, Universidad Andina Simón Bolívar]. Repositorio Institucional. Ecuador. Obtenido de http://hdl.handle.net/10644/3007

Rubiales, M. (2010). Aspectos de la diversidad. Innovación y Experiencias Educativas, $1-9$.

Ruiz, R. (2019). Políticas Y practicas pedagógicas inclusivas para la generación de una cultura (1 ed.). Ibagué: Sello Editorial Universidad del Tolima. Obtenido de http://riut.ut.edu.co/xmlui/bitstream/handle/001/197/RIUT-

Pol\%C3\%ADticas\%20y\%20pr\%C3\%A1cticas\%20pedag\%C3\%B3gicas\%20incl usivas\%20para\%201a\%20generaci\%C3\%B3n\%20de\%20una\%20cultura\%20incl usiva.pdf? sequence $=2 \&$ is Allowed $=\mathrm{y}$

Sampieri, R., Fernández, C., \& Baptista, P. (2016). Metodología de la Investigación. Santa Fe: McGraw-Hill., 546.

Sarrionandía, G., \& Ainscow, M. (2011). La educación inclusiva como derecho.: Marco de referencia y pautas de acción para el desarrollo de una revolución pendiente. Tejuelo: didáctica de la lengua y la literatura. Educación., 26-46.

Watkins, W. (2012). El asalto a la educación pública. Nuevo york; profesores de prensa universitaria.

Zapata, R. (2016). Formación Inicial Docente Universitaria Cultural. Perspectivas desde la región de la Araucania en Chile [Tesis de doctorado, Universidad Autónoma de Barcelona]. Obtenido de https://www.tdx.cat/bitstream/handle/10803/460840/rezp1de1.pdf?sequence=1

\section{Ciencia Ligital}


El artículo que se publica es de exclusiva responsabilidad de los autores y no necesariamente reflejan el pensamiento de la Revista Conciencia Digital.

\section{Liencia}

El artículo queda en propiedad de la revista y, por tanto, su publicación parcial y/o total en otro medio tiene que ser autorizado por el director de la Revista Conciencia Digital.
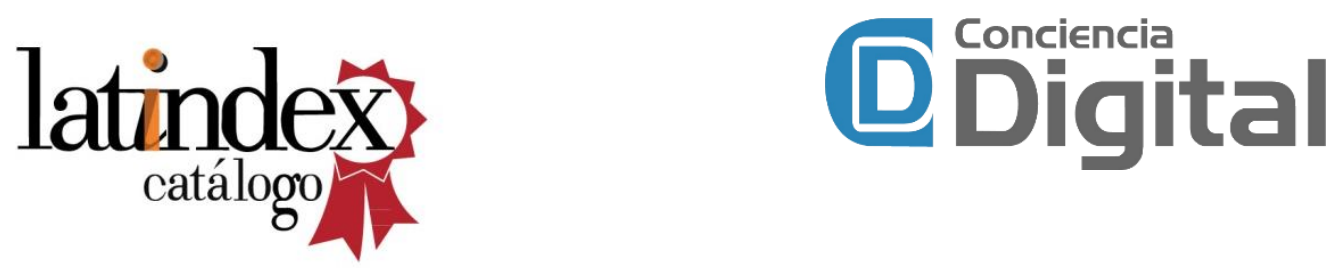

Indexaciones

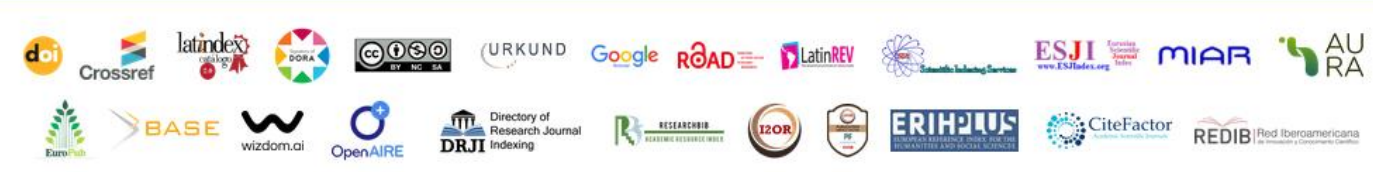

